\title{
Increased physical activity is a cornerstone in the prevention of type 2 diabetes in high-risk individuals. Reply to Laaksonen DE, Lindström J, Tuomilehto J, Uusitupa M [letter]
}

\author{
T. Yates $・$ K. Khunti $\cdot$ F. Bull $・$ T. Gorely $・$ M. Davies
}

Received: 17 August 2007 / Accepted: 20 August 2007 / Published online: 22 September 2007

(C) Springer-Verlag 2007

\section{Abbreviations \\ FDPS Finnish Diabetes Prevention Study}

To the Editor: We thank Laaksonen et al. [1] for raising a number of interesting points. Although we agree with the qualification by Laaksonen et al. regarding our observations on the Da Qing IGT and Diabetes Study [2], this does not alter the conclusions reached in our review. If the selfreported measure of physical activity used in the Da Qing IGT and Diabetes Study [3] gives an accurate representation of physical activity levels, then the large discrepancy in physical activity levels between groups at baseline and the negligible increase in physical activity in the exercise group make the results inconclusive, at least in terms of physical activity. If, however, you accept the flaws inherent with self-reported measures of physical activity and acknowledge that no validity data were provided for the instrument used in the Da Qing IGT and Diabetes Study, then the results from the study in terms of physical activity would again be inconclusive. Either way, the study does not provide good evidence for the effectiveness of physical activity at reducing the risk of diabetes.

T. Yates $(\bowtie) \cdot$ F. Bull $\cdot$ T. Gorely

School of Sport and Exercise Sciences,

Loughborough University, Ashby Road,

Loughborough, Leicestershire LE11 3TU, UK

e-mail: t.e.yates@lboro.ac.uk

K. Khunti

Department of Health Sciences, University of Leicester,

Leicestershire, UK

M. Davies

Department of Cardiovascular Sciences, University of Leicester, Leicestershire, UK
We agree that the success of the Finnish Diabetes Prevention Study (FDPS) in achieving a large decrease in the number of sedentary individuals is encouraging; however, the benefits of this decrease have yet to be quantified. Laaksonen et al. [1] do not agree with our conclusion that the $9 \mathrm{~min} /$ day increase in physical activity found in the FDPS was clinically insignificant. To our knowledge, no randomised-controlled trial has found substantive clinical benefits from an increase in physical activity of this magnitude, independent of other lifestyle changes. Indeed, data from the FDPS found that a difference of twice this magnitude in physical activity change ( $2 \mathrm{~h} /$ week) was not associated with a reduced risk of diabetes after adjusting for other lifestyle variables [4].

Laaksonen et al. [1] question our conclusion that current exercise recommendations may be of insufficient magnitude to reduce the risk of diabetes, independent of other factors. Specifically, they go on to state that at least $2.5 \mathrm{~h} /$ week of walking or moderate-to-vigorous exercise is associated with a decrease in the risk of diabetes. However, $2.5 \mathrm{~h} /$ week of moderate-to-vigorous intensity exercise in the FDPS was not associated with a decreased risk of developing diabetes after adjustment for likely confounders [4]. Furthermore these results were gained by analysing the association of diabetes risk with physical activity levels at follow-up, rather than physical activity change. We accept that the epidemiological evidence for the association between exercise and risk of development of diabetes is well established. However, the key issues for health professionals are whether physical activity change can affect the risk of diabetes independent of other lifestyle factors, such as weight loss, and whether free-living physical activity can 
be successfully promoted in at-risk individuals using costeffective methods. It is these questions that we feel the current evidence from randomised controlled trials is unable to answer.

Laaksonen et al. [1] also question our extrapolation of data from exercise intervention trials in individuals with diabetes to infer that modest increases in physical activity in individuals with pre-diabetes are unlikely to result in weight loss. However, as we highlighted [2], there is growing consensus among national and global health agencies, including the World Health Organization [5], that about $60 \mathrm{~min}$ of moderate intensity exercise on most days of the week is needed to achieve long-term energy balance in the general population [6]. On this basis, although we agree that physical activity can be used to initiate weight loss, we are uncertain whether this can be achieved with the current exercise recommendations of at least $30 \mathrm{~min}$ of moderate intensity exercise on most days of the week. Laaksonen et al. go on to cite unreported data from the FDPS showing that the weight-loss difference between those in the highest and lowest tertiles of moderate-tovigorous exercise change was $2.1 \mathrm{~kg}$ [1]. In fact, there is no disagreement here. Published data from the FDPS show that the difference in median values of moderate-tovigorous physical activity change between those in the lowest and highest tertiles was more than $4 \mathrm{~h} /$ week [4], a value that is substantially higher than the current exercise recommendations. Thus the unreported data from the FDPS do not provide evidence that levels of physical activity that are in line with the current exercise recommendations are sufficient to initiate weight loss.

We agree that there is a need to distinguish between change in $2 \mathrm{~h}$ and fasting glucose values and the risk of developing diabetes, particularly over the longer term, as treatment strategies for diabetes are likely to confound any difference in glucose levels between groups. As we pointed out [2], the area under the glucose curve may be a more sensitive outcome measure than $2 \mathrm{~h}$ and fasting glucose. However, the oral glucose tolerance test is recognised as the gold standard method for assessing glucose control status and $2 \mathrm{~h}$ glucose values have been shown to be a strong predictor of cardiovascular disease risk [7]. We therefore feel that $2 \mathrm{~h}$ glucose is an appropriate main outcome in smaller and/or shorter-term trials on the effect of exercise in those with pre-diabetes.

Publications from the FDPS have made a major contribution to our understanding of the impact of physical activity in reducing the risk of developing diabetes in highrisk populations. The purpose of our review [2] and this response to the letter by Laaksonen et al. [1] was not to cast doubt on the link between physical activity and risk of type 2 diabetes, but to highlight some gaps in the current evidence. We welcome the comments by Laaksonen et al. and hope that continued debate and research into some of the issues identified in our review [2], highlighted in the commentary by Carnethon [8] and challenged in the letter from Laaksonen et al. [1], will help lead to a greater understanding of how physical activity can be utilised to its full potential in helping to out-run the diabetes epidemic.

\section{References}

1. Laaksonen, DE, Lindström J, Tuomilehto J, Uusitupa M (2007) Increased physical activity is a cornerstone in the prevention of type 2 diabetes in high-risk individuals. Diabetologia DOI 10.1007/ s00125-007-0805-y

2. Yates, T, Khunti K, Bull F, Gorely T, Davies MJ (2007) The role of physical activity in the management of impaired glucose tolerance: a systematic review. Diabetologia 50:1116-1126

3. Pan, XR, Li GW, Hu YH et al (1997) Effects of diet and exercise in preventing NIDDM in people with impaired glucose tolerance. The Da Qing IGT and diabetes study. Diabetes Care 20:537-544

4. Laaksonen, DE, Lindström J, Lakka TA et al (2005) Physical activity in the prevention of type 2 diabetes: the Finnish Diabetes Prevention Study. Diabetes 54:158-165

5. World Health Organization (1998) Obesity: preventing and managing the global epidemic. Technical report. WHO, International Obesity Task Force, Geneva

6. Brooks, GA, Butte NF, Rand WM, Flatt JP, Caballero B (2004) Chronicle of the institute of medicine physical activity recommendation: how a physical activity recommendation came to be among dietary recommendations. Am J Clin Nutr 79:921S-930S

7. Coutinho M, Gerstein HC, Wang Y, Yusuf S (1999) The relationship between glucose and incident cardiovascular events. A metaregression analysis of published data from 20 studies of 95,783 individuals followed for 12.4 years. Diabetes Care 22:233-240

8. Carnethon MR (2007) Can we out-run the diabetes epidemic? Diabetologia 50:1113-1115 